\title{
Low Field Magnetic Stimulation (LFMS) on Proliferation and Differentiation of Oligodendrocyte Progenitor Cells
}

\author{
Natasha Dolgova ${ }^{1}$, Zelan Wei ${ }^{1}$, Brandon Spink², QingYun Hua², Le \\ Gui $^{1}$, Zhen Zhang ${ }^{3}$, Xin-Min Li ${ }^{4}$, Yanbo Zhang ${ }^{1,2}$
}

${ }^{1}$ Department of Psychiatry, University of Saskatchewan, Canada; ${ }^{2}$ College of Medicine, University of Saskatchewan, Canada; ${ }^{3}$ Department of Radiology, the First Hospital of China Medical University, China;

\section{INTRODUCTION}

Low Field Magnetic Stimulation (LFMS), a neuromodulation and noninvasive therapy, has shown to produce rapid mood improvement in patients with major depressive disorder ${ }^{(1)}$. Research showed that static magnetic field stimulation enhances oligodendrocyte differentiation by secreting neurotrophic factors ${ }^{(2)}$. Studies showed that increased levels of brain-derived neurotrophic factor and NT-3 are critical for cell proliferation $^{(3,4)}$. Transforming growth factor $\beta$, TGF- $\beta$, known as a multi functional growth factor ${ }^{(5)}$, participates in many essential functions, including cell cycle control, regulation of early development and differentiation, neuron survival and glial differentiation. The underlying molecular mechanisms by which LFMS exerts are remained unclear. Our present data showed that LFMS increased TGF- $\beta 1$ secretion and together with the enhanced differentiation of progenitor cells to oligodendrocytes.

\section{OBJECTIVES}

1) Determine effects of LFMS on proliferation and differentiation of CG4 cells, a bi-potential oligodendrocyte-type-2 astrocyte (O-2A) progenitor cell line, capable of differentiating into either oligodendrocytes or type 2-astrocytes. 2) Investigate mechanisms to explain the differentiation promoting properties of LFMS.

\section{MATERIALS AND METHODS}

1. Cell culture and treatment: LFMS: $20 \mathrm{~min} /$ day at RT with $40 \mathrm{~Hz}$ daily; Sham: $20 \mathrm{~min} /$ day at RT with no magnetic daily; 2 . MTT and WST to measure cell proliferation; 3 . ICC to locate the cell lineage markers; 4. ELISA to measure the cytokine levels in culture medium; 5. Western blot to show the expression levels of cell lineage markers.

\section{RESULTS}
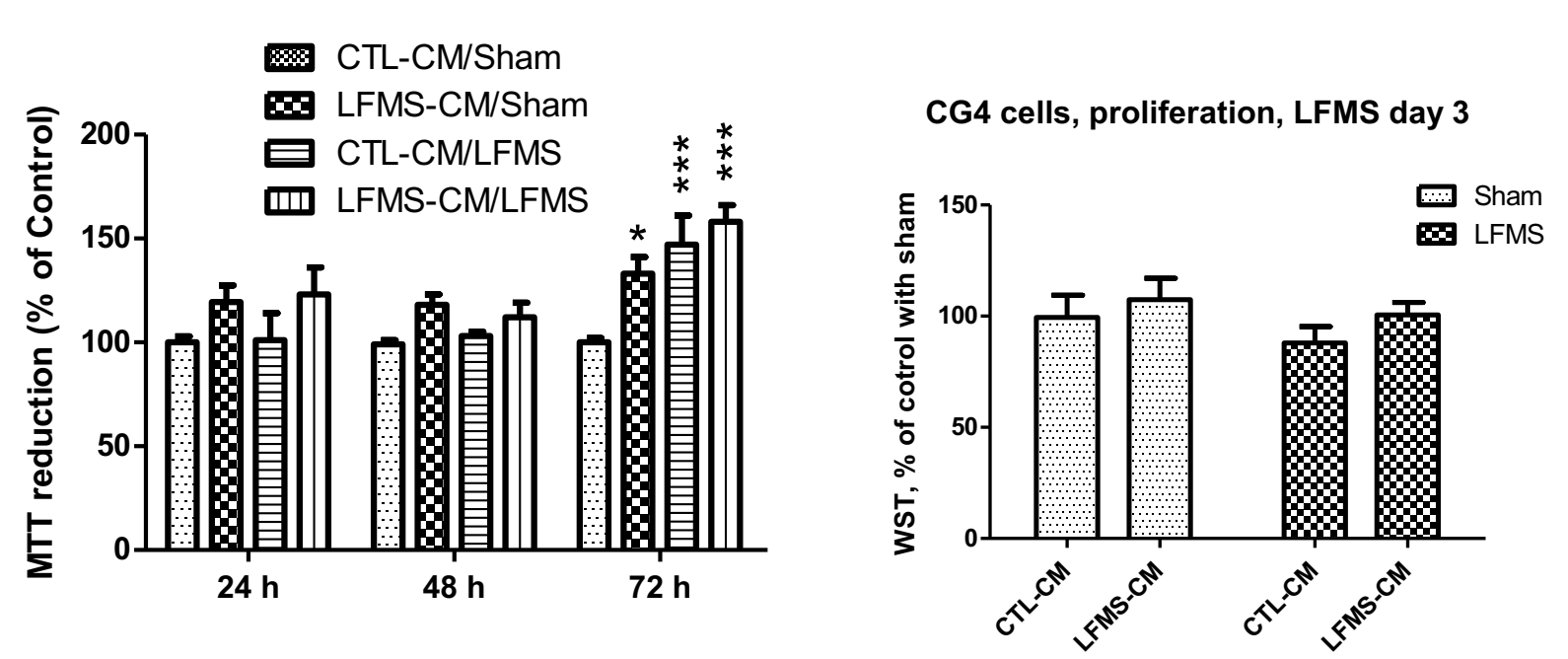
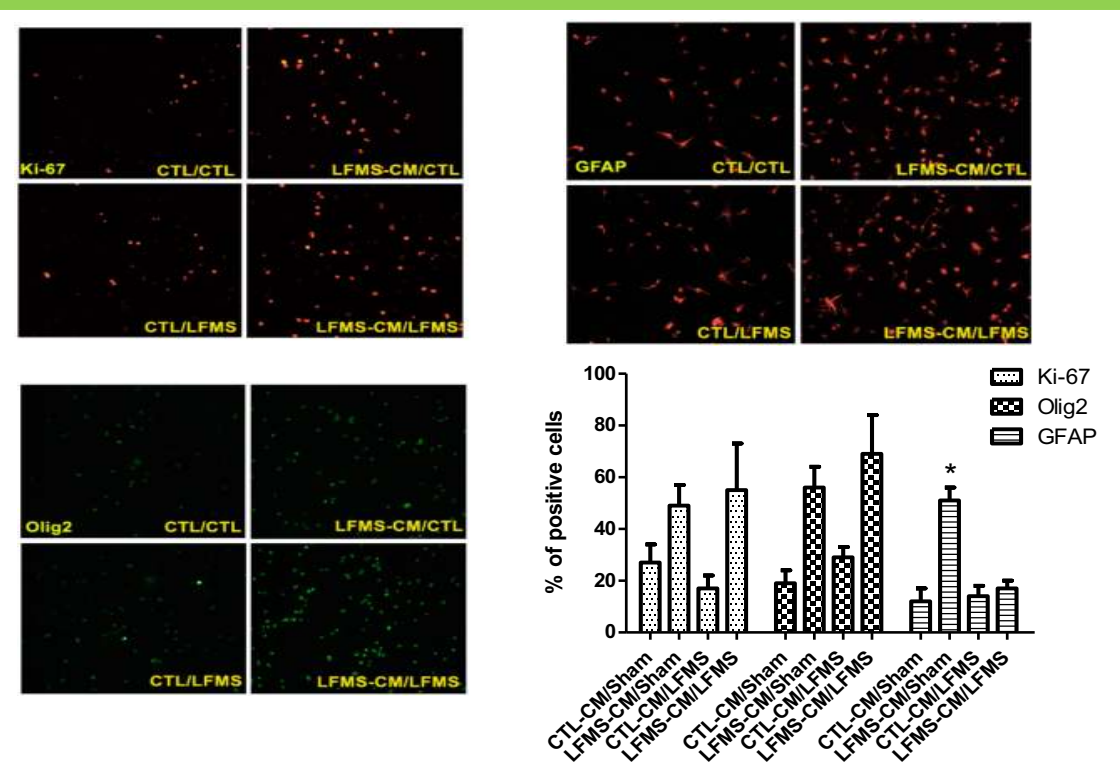

Upper Figure: MTT and WST results showed the CG4 cell proliferation in growth medium (GM, proliferation status) treated with or w/o LFMS. Western blots showed changes of signaling molecules and cell lineage markers. ${ }^{*} p<0.05,{ }^{* * *} p<0.001$ vs corresponding controls. ICC staining showed CG4 cells in proliferation status treated

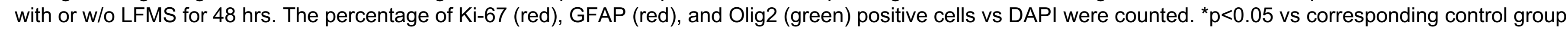
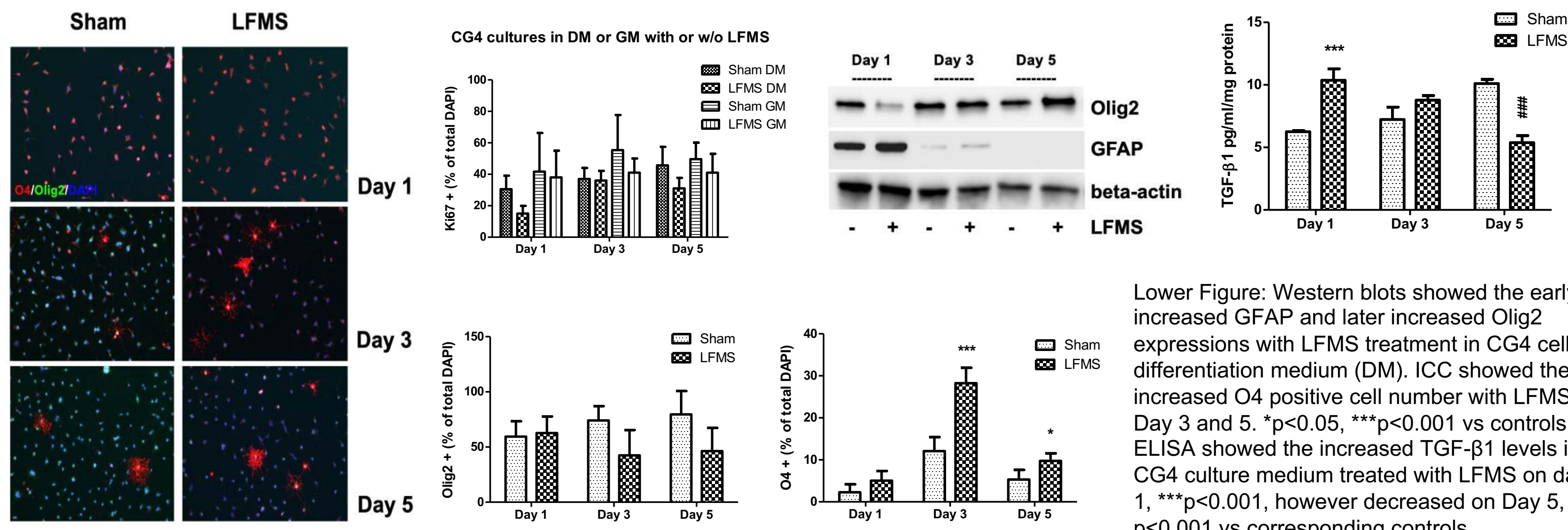

Lower Figure: Western blots showed the early increased GFAP and later increased Olig2
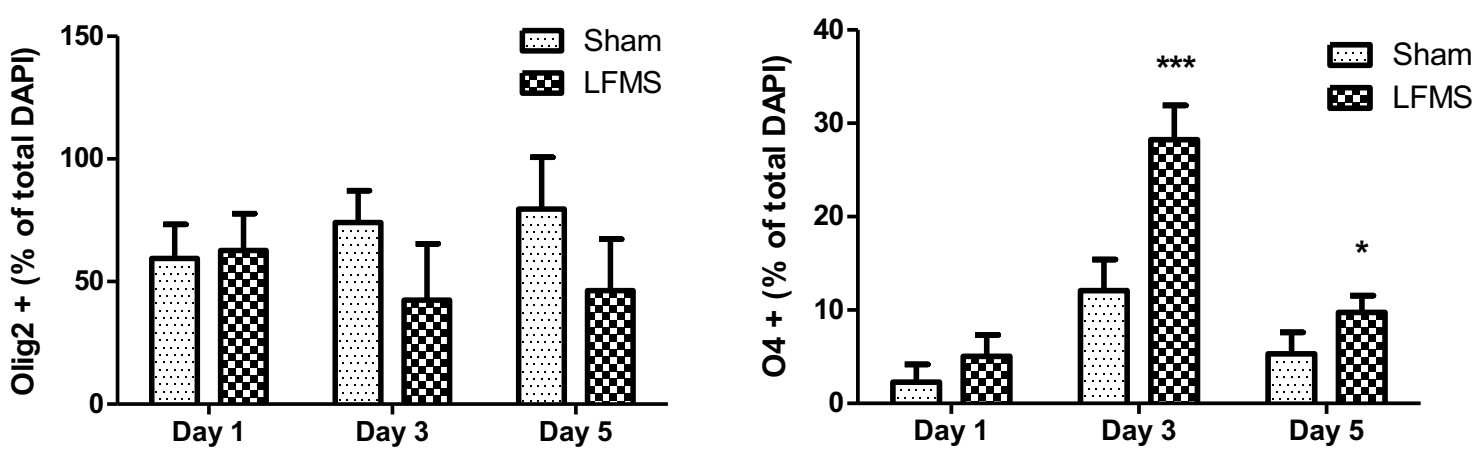
expressions with LFMS treatment in CG4 cells in differentiation medium (DM). ICC showed the increased $\mathrm{O} 4$ positive cell number with LFMS on Day 3 and 5 . ${ }^{*} p<0.05,{ }^{* * *} p<0.001$ vs controls. ELISA showed the increased TGF- $\beta 1$ levels in CG4 culture medium treated with LFMS on day $1,{ }^{* * *} p<0.001$, however decreased on Day $5, \# \#$ $p<0.001$ vs corresponding controls.

\section{Conclusions:}

The present data suggested that: 1) LFMS could preferentially promote differentiation of CG4 (OPCs) cells into oligodendrocyte lineage; 2 ) LFMS increased TGF- $\beta 1$ secretion, when most cells were in astrocyte lineage; TGF- $\beta 1$ secretion was decreased, when cells differentiated to oligodendrocyte lineage. Further work will explore the involvement of TGF- $\beta$ Smad or non-Smad pathways with LFMS treatment.

\section{References}

Rohan ML, et al. Rapid mood-elevating effects of low field magnetic stimulation in depression. Biological psychiatry. 2014 76(3):186 Prasad A, et al. Static Magnetic Field Stimulation Enhance 3. $\mathrm{Xu} \mathrm{H}$, et al. The recovery of hippocampal neurogenesis and BDNF levels, both of which are reduced by repeated restraint stress, is accelerated by chronic venlafaxine. J Pharmacogenomics 2004; 4(5): 322 .

emyelination and neuroinflammation in the brain. 2019 10:332.

Acknowledgement: The authors are grateful for the funding provided by the college of Medicine, University of Saskatchewan start-up fund; Saskatchewan Health Research Fund (SHRF) Establishment grant and Iver and Joyce Graham Indiana Small Professorship. 